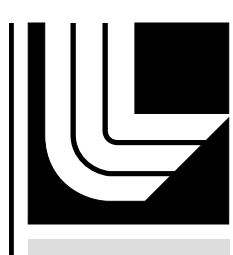

LAW RENCE LIVERMORE N A T IO N A L LABORATORY

\title{
Exp6-polar thermodynamics of dense supercritical water
}

Sorin Bastea, Laurence E. Fried

December 20, 2007

Journal of Chemical Physics 
This document was prepared as an account of work sponsored by an agency of the United States government. Neither the United States government nor Lawrence Livermore National Security, LLC, nor any of their employees makes any warranty, expressed or implied, or assumes any legal liability or responsibility for the accuracy, completeness, or usefulness of any information, apparatus, product, or process disclosed, or represents that its use would not infringe privately owned rights. Reference herein to any specific commercial product, process, or service by trade name, trademark, manufacturer, or otherwise does not necessarily constitute or imply its endorsement, recommendation, or favoring by the United States government or Lawrence Livermore National Security, LLC. The views and opinions of authors expressed herein do not necessarily state or reflect those of the United States government or Lawrence Livermore National Security, LLC, and shall not be used for advertising or product endorsement purposes. 


\title{
Exp6-polar thermodynamics of dense supercritical water
}

\author{
Sorin Bastea* and Laurence E. Fried \\ Lawrence Livermore National Laboratory, \\ P.O. BOX 808, Livermore, CA 94550
}

\begin{abstract}
We introduce a simple polar fluid model for the thermodynamics of dense supercritical water based on a Buckingham (exp-6) core and point dipole representation of the water molecule. The proposed exp6-polar thermodynamics, based on ideas originally applied to dipolar hard spheres, performs very well when tested against molecular dynamics simulations. Comparisons of the model predictions with experimental data available for supercritical water yield excellent agreement for the shock Hugoniot, isotherms and sound speeds, and are also quite good for the self-diffusion constant and relative dielectric constant. We expect the present approach to be also useful for other small polar molecules and their mixtures.
\end{abstract}

PACS numbers:

*Electronic address: sbastea@llnl.gov 
The properties of water remain a source of much interest and fascination for scientists and non-scientists alike [1]. This is certainly due in part to its crucial role in the development and evolution of biological, i.e. live, systems, but understanding the behavior of water at a fundamental level is also of major importance to geoscience, environmental sciences, planetary modeling and numerous industrial applications. For many of the latter circumstances the relevant thermodynamic regimes include pressures and temperatures much higher than ordinarily encountered. Examples of such "extreme" states of water can be found in problems ranging from the physics and chemistry of the giant planets [2], dynamics of the Earth's mantle [3] and origin of the Earth's hydrocarbons [4], to supercritical water oxidation technologies [5]. The breadth of the experimental research efforts covering the equilibrium (and more recently nonequilibrium [6]) properties of water at high pressures and temperatures is too large to be detailed here. On the theoretical side simulations based on a variety of models [7] continue to provide important insights into the behavior of water under a wide range of thermodynamic conditions, while empirical equations of state $[8,9]$ help guide both practical applications and modeling efforts. In the last decade molecular dynamics simulations based on the density functional theory (DFT) have also contributed significantly to our understanding of water[10, 11]. Nevertheless, classical empirical models of water remain very successful in capturing its behavior under a broad spectrum of conditions. These models, which usually assume a rigid geometry, point charges and no polarizability $[12,15]$ remain much studied [17] due to their effectiveness and versatility; enhanced variants of this basic motif have also been considered [16]. Despite their simplicity such representations of the water molecule are still too complicated to be efficiently analyzed with the tools of statistical mechanics. As a result, water equations of state are usually not based on the microscopics of the water molecule, but typically are highly parametrized fits of experimental results, simulation results or both. Previous efforts to construct equations of state that more directly account for the molecular properties of water have sometimes relied on spherical averaging, leading to temperature dependent potentials [18], or representations of the associative character of the water molecule based on multiple species [19]. Unfortunately polar interactions are not amenable to useful spherical approximations [20], while multi-species models, although practical, are rather ad hoc.

In the present paper we wish to narrow the disconnect between the molecular properties of water and its fluid thermodynamics, particularly in the supercritical region, and therefore 
to facilitate accurate and fast equation of state predictions for water (and ultimately water mixtures) in an extensive domain of pressures and temperatures. Even though our model is necessarily limited in its scope - it does not account for example for the dissociation observed in highly compressed water - we expect it to be useful up to significantly high pressures and temperatures, roughly bounded by $15 \mathrm{GPa}$ and $2000 \mathrm{~K}$.

To this end we propose a representation of the water molecule consisting of a Buckingham exp-6 core and a central point dipole. This is certainly a much simplified picture, but one that we believe preserves the molecular features that are likely to be most important at supercritical conditions. This representation is in fact not very different than typical water models such as SPC [12] and its later versions, which assume van der Waals type interactions between the oxygens and place partial charges on both the oxygen and the point hydrogens, thereby yielding the expected dipole moment. Our description preserves the polar character of the water molecule and its effect on long distance interactions, as well as some, but clearly not all the short range electrostatic correlations. After all, hydrogen bonding in water leads to the formation of a tetrahedral network which cannot be reproduced by point dipole interactions alone. Nevertheless, at high densities and temperatures the short range repulsive interactions are known to play the dominant role, e.g. in typical simple liquids, and despite its complexity water appears to be no exception $[21,22]$. Therefore, in a significant domain of supercritical conditions the electrostatic interactions in water can likely be treated as perturbations acting on the structure determined by the strong oxygen-oxygen repulsions, and the point dipole description is then the simplest approximation. Our view of supercritical water is thus that of a simple polar fluid, similar with the fluids originally considered by Stell and coworkers $[23,24]$. Their analysis of the quintessential simple polar fluid - dipolar hard spheres - showed that its thermodynamics is very well described by a perturbation theory that uses the hard spheres fluid as a reference, particularly when employing a Padé approximant summation of the perturbation series. Additional approximations also yield an analytical description of the thermodynamics of the polar fluid with a Lennard-Jones core $[23,25,26]$, i.e. Stockmayer fluid, employed for example in [27].

Our water model on the other hand uses an exp-6 core,

$$
u(r)=\epsilon\left[A e^{-\alpha \frac{r}{r_{0}}}-B\left(\frac{r_{0}}{r}\right)^{6}\right]
$$

$A=6 e^{\alpha} /(\alpha-6), B=\alpha /(\alpha-6)$, which is known to be effective in the modeling of hot, dense 
non-polar fluids [28]. This potential has also been shown to be superior to the Lennard-Jones one as a representation of the the oxygen-oxygen interaction in water [15]. Our approach to the thermodynamics of this system is detailed below.

Let $f=F / N k_{B} T$ be the excess reduced Helmholtz free energy. Then, treating the dipole-dipole interaction as a perturbation, $f$ can be expanded in powers of $\mu^{2}(\mu$ - dipole moment):

$$
f=f_{0}+f_{2}+f_{3}+\ldots=f_{0}+\Delta f_{d}
$$

where $f_{0}$ corresponds to the reference exp-6 fluid and $f_{1}$ vanishes [24].

For the calculation of $f_{0}$ we employ the Mansoori-Canfield variational method [29] as augmented by Ross [30]:

$$
f_{0}(\rho, T) \leq f_{h s}(\eta)+12 \eta \beta \int_{1}^{\infty} s^{2} g_{h s}(s ; \eta) u(\sigma s) d s+\Delta f_{\text {Ross }}(\eta)
$$

, where $\eta=\pi \rho \sigma^{3} / 6, f_{h s}(\eta)$ and $g_{h s}(s ; \eta)$ are the excess free energy and pair-correlation function of the hard spheres system, respectively, and $\Delta f_{\text {Ross }}(\eta)$ is the Ross correction. $f_{0}(\rho, T)$ is then determined by the minimum of the right-hand side with respect to the hard sphere diameter $\sigma$, attained for a value $\sigma_{0}$.

Following Stell et al. [24] we would now like to calculate the polar contribution $\Delta f_{d}$ by using a Padé summation of the dipole perturbation series, $\Delta f_{d}=f_{2} /\left(1-f_{3} / f_{2}\right)$. Unfortunately the determination of $f_{2}$ and $f_{3}$ requires the 2-particle and 3-particle correlation functions of the reference system [24], which are unavailable. To make further progress we use therefore the hard spheres fluid with diameter $\sigma_{0}$ as a reference, instead of the exp-6 fluid. This allows us to employ the analytical representations available for the hard spheres system. Explicitly, we use

$$
\begin{aligned}
& f_{2}==-\frac{\rho^{*} \beta_{d}^{2} I_{6}^{h s}\left(\rho^{*}\right)}{6} \\
& f_{3}=\frac{\rho^{* 2} \beta_{d}^{3} I_{d d d}^{h s}\left(\rho^{*}\right)}{54}
\end{aligned}
$$

, where $\rho^{*}=\rho \sigma_{0}^{3}, \beta_{d}=\mu^{2} / k_{B} T \sigma_{0}^{3}$, and $I_{6}^{h s}, I_{d d d}^{h s}$ are pair and triple integrals of the dipole perturbation with the hard sphere fluid distribution functions, which have been accurately parameterized in [31].

Equations $2-4$ then yield the full thermodynamics of the proposed exp6-polar water model, and we wish to test its predictions using molecular dynamics simulations. Accordingly, we performed such simulations using 500 to 1372 molecules in the $N V T$ ensemble, at 
temperatures $T=720 \mathrm{~K}, 1400 \mathrm{~K}$, and $2000 \mathrm{~K}$, and densities up to $1.8 \mathrm{~g} / \mathrm{cc}$. The electrostatic interactions were handled using the Ewald summation technique with conducting boundary conditions. The potential parameters used were $\epsilon / k_{B}=222.3 K, r_{0}=3.535 \AA, \alpha=11.47$ and dipole moment $\mu=2.188 D$. (Mass was $m=18 m_{0}$ and moment of inertia $I=1.25 m_{0} \AA^{2}$; $m_{0}$ - atomic mass unit.) The length of the runs was $50-100 \mathrm{~ns}$.

The calculated thermodynamic quantities included the standard ones, e.g. pressure and energy, as well as the self-diffusion coefficient $D$ and relative dielectric constant $\epsilon_{r}$ of the system. $D$ was calculated using the familiar velocity autocorrelation relation:

$$
D=\lim _{t \rightarrow \infty} \frac{1}{3} \int_{0}^{t}\left\langle\mathbf{v}_{i}(0) \cdot \mathbf{v}_{i}(\tau)\right\rangle d \tau
$$

with no tail corrections. The dielectric constant is related by linear response theory to the fluctuations of the dipole moment of the system, $\mathbf{M}=\sum_{i} \mu_{i}$ [32]. For conducting boundary conditions the result is

$$
\epsilon_{r}=1+\frac{4 \pi}{3} \frac{\left\langle\mathbf{M}^{2}\right\rangle}{V k_{B} T}
$$

, which can also be written as $\epsilon_{r}=1+3 y g$, with $y=4 \pi \rho \mu^{2} / 9 k_{B} T$ a dimensionless parameter and $g=\left\langle\mathbf{M}^{2}\right\rangle / N \mu^{2}$ the Kirkwood correlation factor. Such fluctuations-based estimates typically require very long runs to achieve reasonable accuracy, which is the reason our simulations extended well into the tens of nanoseconds duration.

To make theoretical predictions for $D$ we turn to entropy scaling ideas [33-36], connecting the self-diffusion constant (and in particular its variation relative to the low density limit $[35,36])$ to the excess entropy of the system. Since results for the diffusion constant at low densities are not available for the present exp6-polar system (or the dipolar hard spheres), we simply calculate $D$ for the reference exp-6 fluid using the variational effective hard sphere diameter $\sigma_{0}[35,36]$ :

$$
\frac{D}{D_{B} \chi}=\exp \left(\gamma s_{e} / k_{B}\right)
$$

$D_{B}$ is the low density (Boltzmann) diffusion coefficient, $\chi$ is the contact value of the hard spheres pair correlation function, $s_{e}$ is the excess entropy and $\gamma=0.8$ a numerical constant. For the dielectric constant we employ the dipolar hard spheres perturbation theory of Tani et al. [37]

$$
\epsilon_{r}=1+3 y+3 y^{2}+3 y^{3}\left[\frac{9 I_{d d \Delta}\left(\rho^{*}\right)}{16 \pi^{2}}-1\right]
$$

again using the effective hard sphere diameter $\sigma_{0}$. 
The simulation results are compared with theoretical predictions in Figs. 1 and 2. Obviously as far as equation of state calculations are concerned the agreement between simulations and theoretical predictions - Eqs. 2-4 - is very good for the energy, and even better for the pressure. Moreover, the predictions for $D$ and $\epsilon_{r}$ also appear to agree very well with simulations. The larger deviations observed for the dielectric constant at low temperatures and high densities are likely due to the system approaching freezing. The very good quality of the agreement between the diffusion coefficient calculated in the simulations and the entropy scaling result is somewhat surprising, since the prediction, as noted before, is actually for the reference exp-6 system, i.e. without the dipole. This suggests therefore that at the thermodynamic conditions of interest here the effect of the point dipole on self-diffusion is rather small and that "caging" effects remain dominant.

Since the testing of the proposed thermodynamics has been successful, we would like to compare it now with available experimental data for supercritical water. Predicting thermodynamic properties in this regime has been our clearly stated goal. Nevertheless, although beyond the scope of our present undertaking, we start with a comparison between the experimentally determined structure of water and that found in the MD simulations. We show in Fig. 3 the structure factor at $T=723 \mathrm{~K}$ and $P=0.1 G P a$, as measured in Ref. [38], and the MD result at $T=720 \mathrm{~K}$ and $P=0.13 G P a$. The agreement is essentially only qualitative, as expected for the model employed here and the low temperature and pressure involved. Better agreement is however possible and likely at higher densities and temperatures, where the role of hydrogen bonding should further decrease.

In Fig. 4 we show comparisons of the model predictions with the water shock Hugoniot [39], isotherms of Withers et al. [40], and sound speed data of Abramson and Brown [41], all indicating excellent agreement. Moreover, the model estimates for the critical density and temperature of water are $\rho_{c} \simeq 0.32 \mathrm{~g} / \mathrm{cc}$ and $T_{c} \simeq 710 \mathrm{~K}$, which compare at least as well as other models (e.g. for SPC water $\rho_{c} \simeq 0.27 \mathrm{~g} / c c$ and $T_{c} \simeq 594 K$ ) with the experimental values $-\rho_{c}^{e x p}=0.322 \mathrm{~g} / c c, T_{c}^{e x p}=647.1 \mathrm{~K}$. The comparison with available self-diffusion and dielectric constant data - Fig. 5, is not as accurate as for the equation of state, but the predictions remain very meaningful.

In conclusion, we introduced a new model for the thermodynamics of dense supercritical water based on a Buckingham (exp-6) core and point dipole representation of the water molecule. The present paradigm reproduces very well equation of state type experimental 
data available, including Hugoniot, isotherms and sounds speeds, and in addition produces very satisfactory predictions for the self-diffusion constant and relative dielectric constant. This approach should prove useful for other small polar molecules as well, e.g. $\mathrm{NH}_{3}, \mathrm{HF}$, $\mathrm{HCl}$ etc., and can be easily generalized to polar-nonpolar mixtures. As a result, it should be particularly useful for complex chemical equilibrium calculations, such as the ones performed in Ref. [4] or those typically encountered in planetary or detonation modeling.

This work was performed under the auspices of the U. S. Department of Energy by Lawrence Livermore National Laboratory under Contract DE-AC52-07NA27344.

[1] N. Angier, Small, Yes, but Mighty: The Molecule Called Water, The New York Times, July $10(2007)$.

[2] W.B. Hubbard, Science 275, 1279 (1979).

[3] M.M. Hirschmann, Annu. Rev. Earth Planet. Sci. 34, 629 (2006).

[4] H.P. Scott, R.J. Hemley, H.K. Mao, D.R. Herschbach, L.E. Fried, W.M. Howard, S. Bastea, Proc. Natl. Acad. Sci. USA 101, 14023 (2004).

[5] H. Weingärtner, E.U. Franck, Angew. Chem. Int. Ed. 44, 2672 (2005).

[6] M. Bastea, S. Bastea, J.E. Reaugh, D.B. Reisman, Phys. Rev. B 75, 172104 (2007).

[7] See, e.g., B. Guillot, J. Mol. Liq. 101, 219 (2002).

[8] K.S. Pitzer, S. M. Sterner, J. Chem. Phys. 101, 3111 (1994).

[9] W. Wagner, A. Prub, J. Phys. Chem. Ref. Data 31, 387 (2002).

[10] N. Goldman, L.E. Fried, I.F.W. Kuo, C.J. Mundy, Phys. Rev. Lett. 94, 217801 (2005).

[11] E. Schwegler, G. Galli, F. Gygi, R.Q. Hood, Phys. Rev. Lett. 87, 265501 (2001).

[12] F.H. Stillinger, A. Rahman, J. Chem. Phys. 60, 1545 (1974).

[13] W.L. Jorgensen, J. Chandrasekhar, J.D. Madura, R.W. Impley, M.L. Klein, 79, 926 (1983).

[14] H.J.C. Berendsen, J.R. Grigera, T.P. Straatsma, J. Phys. Chem. 91, 6269 (1987).

[15] J.R. Errington, A.Z. Panagiotopoulos, J. Phys. Chem. B 102, 7470 (1998).

[16] B. Guillot, Y. Guissani, J. Chem. Phys. 114, 6720 (2001).

[17] J.L.F. Abascal, C. Vega, Phys. Rev. Lett. 98, 237801 (2007).

[18] F.H. Ree, J. Chem. Phys. 76, 6287 (1982).

[19] L.E. Fried, W.M. Howard, J. Chem. Phys. 110, 12023 (1999). 
[20] D. MacGowan, D.B. Nicolaides, J.L. Lebowitz, and C.-K. Choi, Mol. Phys. 58, 131 (1986).

[21] Th. Strässle, A.M. Saitta, Y. Le Godec, G. Hamel, S. Klotz, J.S. Loveday, R.J. Nelmes, Phys. Rev. Lett. 96, 067801 (2006).

[22] A.F. Goncharov, C. Sanloup, N. Goldman, J.C. Crowhurst, S. Bastea, L.E. Fried, N. Guignot, M. Mezouar, Y. Meng, submitted to Proc. Natl. Acad. Sci. USA.

[23] G. Stell, J.C. Rasaiah, H. Narang, Mol. Phys. 23, 393 (1972).

[24] G.S. Rushbrooke, G. Stell, J.S. Høye, Mol. Phy. 26, 1199 (1973).

[25] K.E. Gubbins, C.H. Twu, Chem. Eng. Sci. 33, 863 (1977).

[26] C.H. Twu, K.E. Gubbins, Chem. Eng. Sci. 33, 879 (1977).

[27] S.V. Churakov, M. Gottschalk, Geochim. Cosmochim. Ac. 67, 2397 (2004).

[28] M. Ross, F.H. Ree, J. Chem. Phys. 73, 6146 (1980).

[29] J.A. Barker, D. Henderson, Rev. Mod. Phys. 48, 587 (1976).

[30] M. Ross, J. Chem. Phys. 71, 1567 (1979).

[31] B. Larsen, J.C. Rasaiah, G. Stell, Mol. Phys. 33, 987 (1977).

[32] J.-P. Hansen, I.R. McDonald, Theory of Simple Liquids, $2^{\text {nd }}$ edition, (Academic Press, London, 1986).

[33] Y. Rosenfeld, Phys. Rev. A 15, 2545 (1977).

[34] M. Dzugutov, Nature(London) 381, 137 (1996).

[35] S. Bastea, Phys. Rev. E. 68, 031204 (2003).

[36] S. Bastea, Phys. Rev. Lett. 93, 199603 (2004).

[37] A. Tani, D. Henderson, J.A. Barker, C.E. Hecht, Mol. Phys. 48, 863 (1983).

[38] Y.E. Gorbatyi, Y.N. Demyanets, J. Struct. Chem. 23, 882 (1982).

[39] S. P. Marsh (Ed.), LASL Shock Hugoniot Data, (Univ. California Press, Berkeley, 1980).

[40] A.C. Withers, S.C. Kohn, R.A. Brooker, B.J. Wood, Geochim. Cosmochim. Ac. 64, 1051 (2000).

[41] E.H. Abramson, J.M. Brown, Geochim. Cosmochim. Ac. 68, 1827 (2004).

[42] W.J. Lamb, G.A. Hoffman, J. Jonas, J. Chem. Phys. 74, 6875 (1981).

[43] K. Heger, M. Uematsu, E.U. Frank, Ber. Bunsenges. Phys. Chem. 84, 758 (1980). 


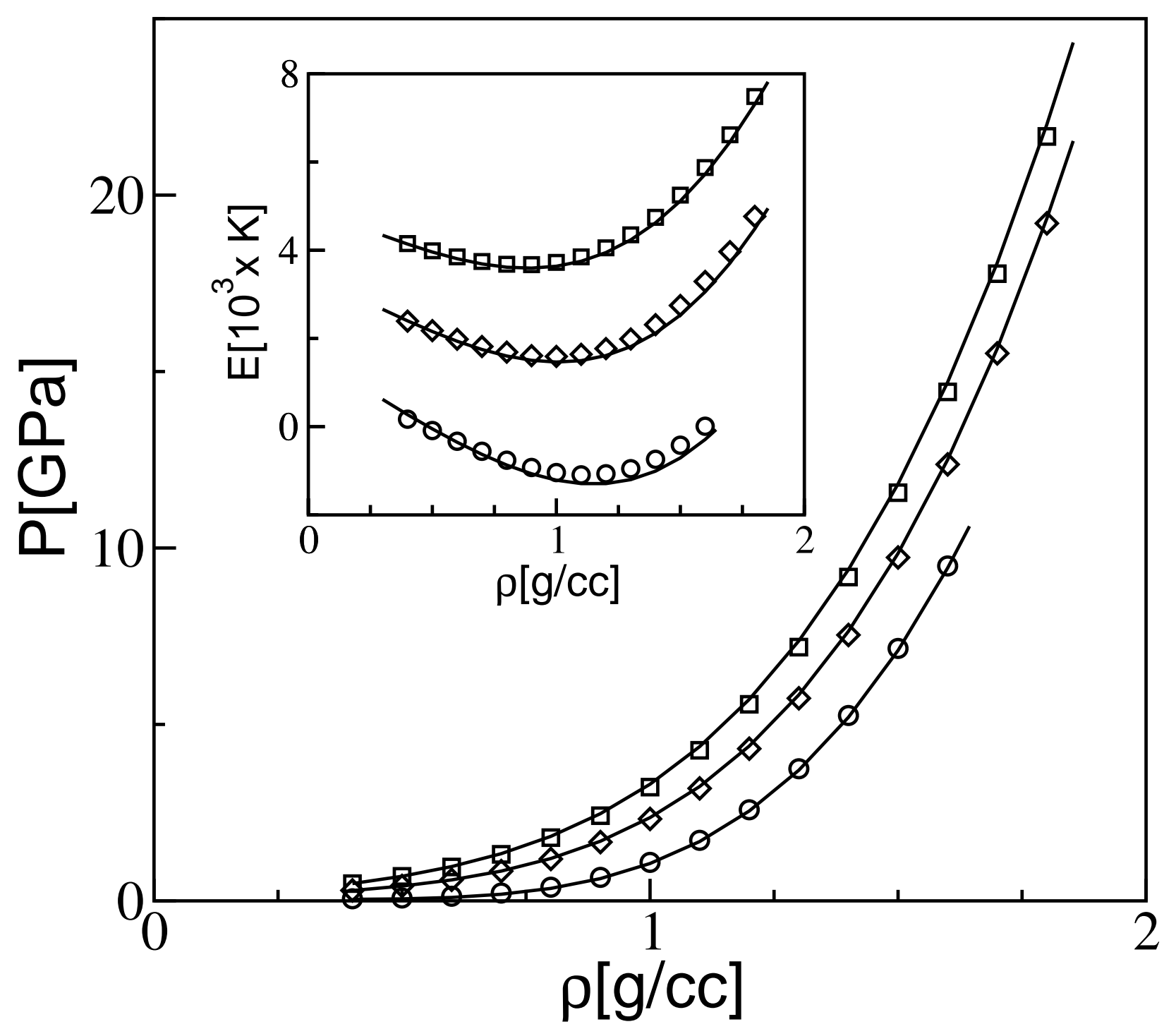

FIG. 1: Comparison of simulation results (symbols) and predicted thermodynamics (lines) (relations 2 - 4 in the text); $T=720 K$ (circles), $1400 K$ (diamonds), and $2000 K$ (squares). 


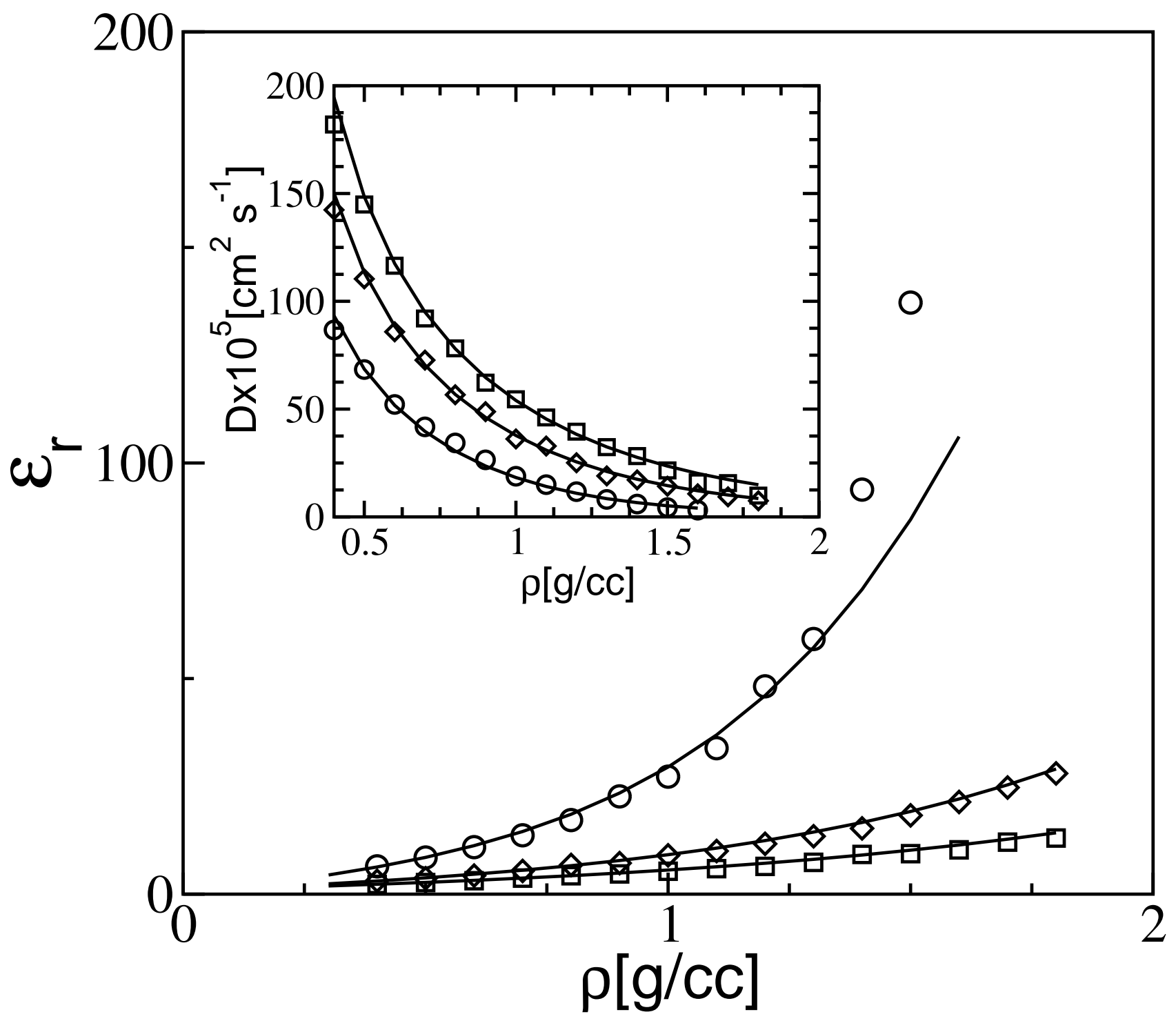

FIG. 2: Comparison of simulation results (symbols) and theoretical predictions (lines) (relation 7 for self-diffusion constant $D$ and 8 for relative dieletric constant $\left.\epsilon_{r}\right) ; T=720 K$ (circles), $1400 K$ (diamonds), and $2000 K$ (squares). 


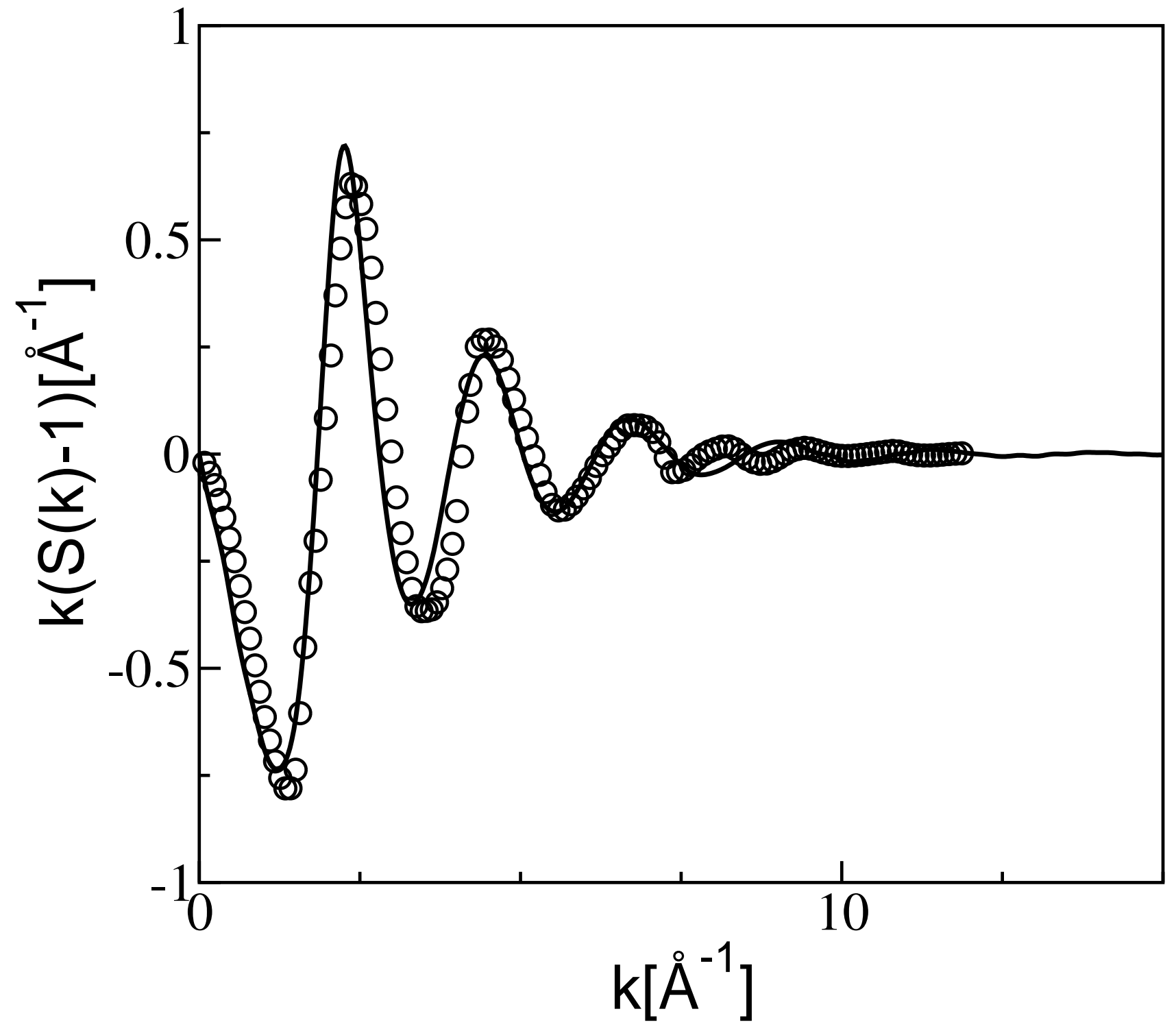

FIG. 3: Experimental strucure factor of water at $T=723 \mathrm{~K}$ and $P=0.1 G P a[38]$ (symbols) and MD simulations result at $T=720 K$ and $P=0.13 G P a$ (line). 


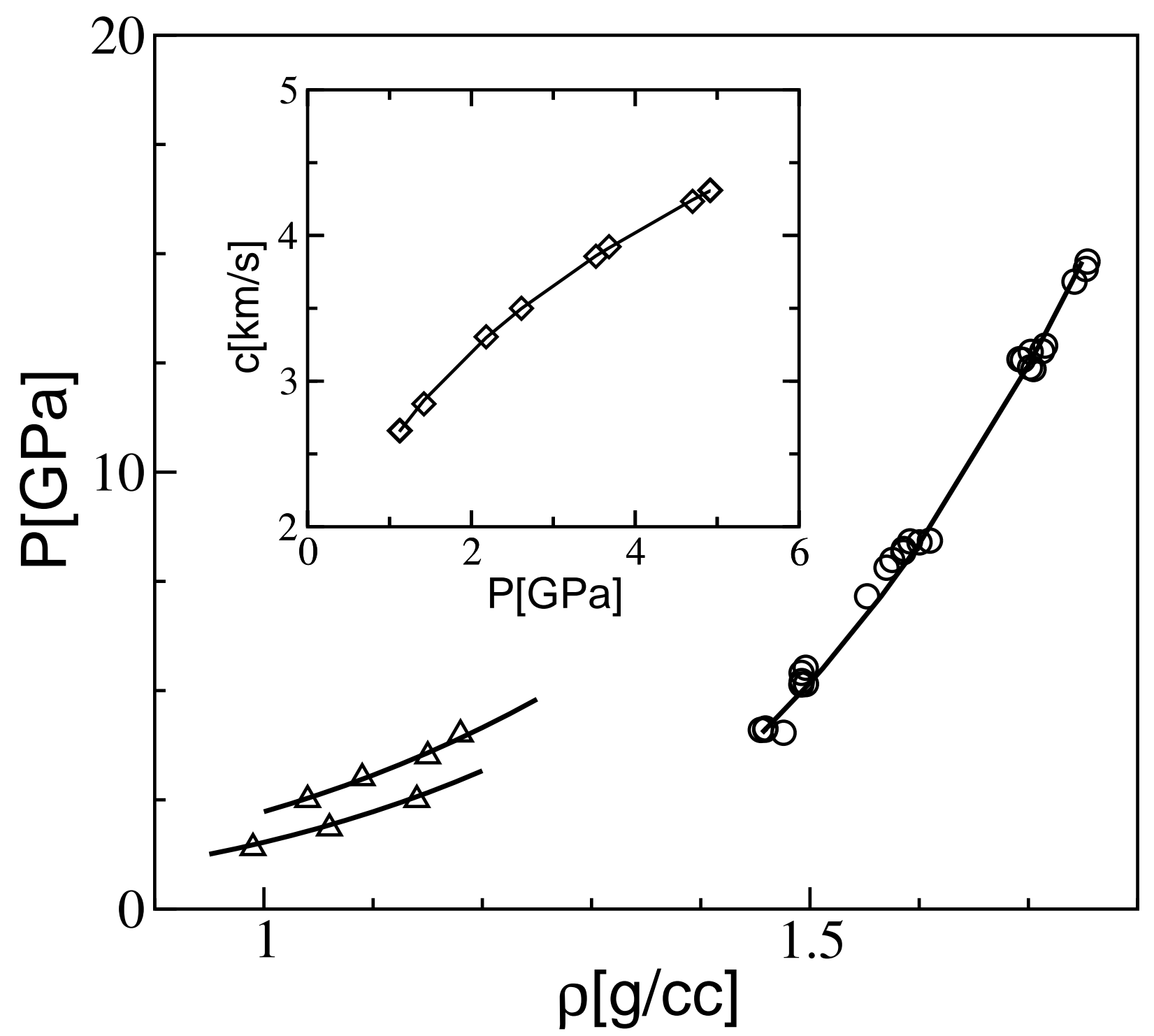

FIG. 4: Comparison of experimental results (symbols) and proposed water thermodynamics (relations $2-4$ with $\epsilon / k_{B}=222.3 K, r_{0}=3.535 \AA$, $\alpha=11.47, \mu=2.188 D$ ). Water Hugoniot (circles) Ref. [39], $T=983 K$ and $1373 K$ isotherms (triangles) - Ref. [40], sound speed at $T=673 K$ (inset - diamonds) - Ref. [41]. 


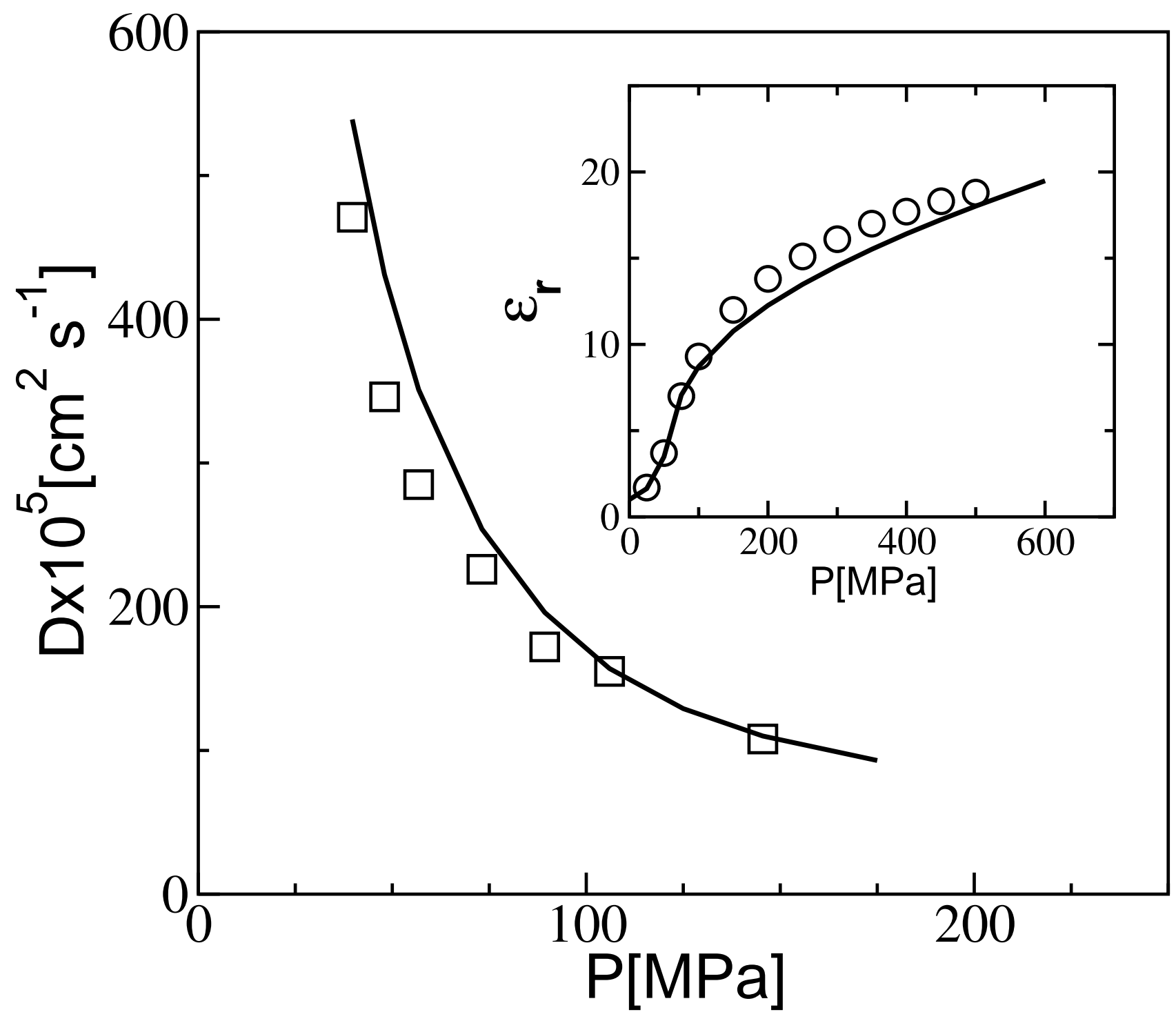

FIG. 5: Comparison of experimental data (symbols) and predicted values (lines) (relation 7 for self-diffusion constant $D$ and 8 for $\epsilon_{r}$ ). Self-diffusion constant $D$ at $T=973 K$ (squares) - Ref. [42], and relative dieletric constant $\epsilon_{r}$ at $T=773 K$ - Ref. [43]. 